Original Article

\title{
Heterostructure of Titanium Dioxide Nanotube/Graphene Nanoplatelet with Enhancing Photocatalytic Activity
}

\author{
Vo Quang Mai, Vo Chi Hao, Nguyen Huu Tho, Nguyen Xuan Sang* \\ Saigon University, 273 An Duong Vuong, ward 3, district 5, Ho Chi Minh City, Vietnam
}

Received 18 September 2019

Revised 15 April 2020; Accepted 01 August 2020

\begin{abstract}
In this study, the composite of titanium dioxide nanotube (TNT) and graphene nanoplatelet was hydrothermally synthesized in a sodium hydroxide solution. Morphological and crystalline properties of the synthesized samples were analyzed by transmission electron microscopy (TEM), X-ray differactometry (XRD), and Raman spectroscopy. Optical properties were investigated by UV-Vis diffuse reflextance spectra (DRS) where optical bandgap was determined. Photocatalytic activity of the synthesized samples was evaluated through the degradation of methylene blue in the solution under direct sunlight irradiation. The result showed the enhancement of photocatalytic activity in the composite sample in compare to the bare TNT. After $120 \mathrm{~min}$ of irradiation, the photocatalytic efficiency of the composite and TNT was $~ 95 \%$ and $63 \%$, respectively. Mechanism of enhanced activity was supported by DRS measurements in which the composite showed the higher visible light absoption and lower bandgap value. Optical bandgap of the $\mathrm{sGr} / \mathrm{TNT}$ composite was about $3.25 \mathrm{eV}$ which was notably reduced in compare to that of the bare TNT of $\sim 3.68 \mathrm{eV}$.
\end{abstract}

Keywords: titanium nanotube, graphene, heterostructure, photocatalysis, DRS.

\footnotetext{
* Corresponding author.

Email address: sangnguyen@sgu.edu.vn 


\title{
Cấu trúc dị thể của tổ hợp ống nano $\mathrm{TiO}_{2} /$ graphene nano dạng đĩa với sự tăng cường khả năng xúc tác quang
}

\author{
Võ Quang Mai, Võ Chí Hào, Nguyễn Hữu Thọ, Nguyễn Xuân Sáng* \\ Truờng Đại học Sài Gòn, 273 An Duoong Vuơng, Phuờng 3, Quận 5, Thành phố Hồ Chí Minh, Việt Nam \\ Nhận ngày 18 tháng 9 năm 2019 \\ Chỉnh sửa ngày 15 tháng 4 năm 2020; Chấp nhận đăng ngày 01 tháng 8 năm 2020
}

\begin{abstract}
Tóm tắt: Trong nghiên cứu này, vật liệu ống nano $\mathrm{TiO}_{2}$ (TNT) tổ hợp với các tấm nano graphene (Gr) dạng đĩa (nanoplatelete) được chế tạo bằng phương pháp thủy nhiệt trong môi trường kiềm. Hình thái bề mặt và các đặc tính của vật liệu được phân tích bằng hình ảnh hiển vi điện tử truyền qua (TEM), nhiễu xạ tia X (XRD) và phổ dao động Raman. Tính chất quang của vật liệu được phân tích bằng phổ UV-Vis phản xạ rắn (DRS). Khả năng xúc tác quang của vật liệu được khảo sát bằng sự suy giảm màu của dung dịch methylene xanh dưới ánh nắng mặt trời trực tiếp, kết quả cho thấy khả năng xúc tác quang vượt trội của vật liệu tổ hợp với hiệu suất lên đến $~ 95 \%$, trong khi vật liệu TNT $63 \%$. Cơ chế tăng cường khả năng xúc tác quang được giải thích bằng sự tăng cường sự hấp thụ vùng ánh sáng khả kiến cùng với sự suy giảm bề rộng vùng cấm ở vật liệu tổ hợp thông qua phổ DRS. Cụ thể, năng lượng vùng cấm của vật liệu tổ hợp sGr/TNT $3.25 \mathrm{eV}$ giảm đáng kể so với TNT $\sim 3.68 \mathrm{eV}$.
\end{abstract}

Từ khóa: ống nano $\mathrm{TiO}_{2}$, graphene, cấu trúc dị thể, xúc tác quang, DRS.

\section{Mở đầu}

Gần đây, khả năng quang xúc tác vùng ánh sáng khả kiến của các vật liệu bán dẫn nhằm xử lý chất thải từ ngành công nghiệp dệt nhuộm đang nhận được nhiều sự quan tâm bởi các nhà nghiên cứu trên toàn thế giới. Các chất bán dẫn oxit như $\mathrm{TiO}_{2}, \mathrm{ZnO}, \mathrm{CdS}, \mathrm{WO}_{3}$ dều thể hiện được tính chất xúc tác quang, đặc biệt là $\mathrm{TiO}_{2}$ với những ưu điểm như: chi phí thấp, an toàn sinh học, độ bền và hoạt tính quang hóa cao [1]. Tuy nhiên, tốc độ tái hợp của cặp điện tử và lỗ trống $\left(\mathrm{e}^{-} / \mathrm{h}^{+}\right)$của $\mathrm{TiO}_{2}$ nhanh làm giảm hiệu quả xúc tác quang, đồng thời năng lượng vùng cấm lớn $(>3.0 \mathrm{eV})$ nên cần nguồn ánh sáng kích thích nằm trong vùng tử ngoại (UV) [2]. $\mathrm{TiO}_{2}$ dạng

\footnotetext{
* Tác giả liên hệ.

Địa chỉ email: sangnguyen@sgu.edu.vn
}

https://doi.org/10.25073/2588-1140/vnunst.4947 ống lần đầu tiên được chế tạo bởi Hoyer năm 1996, hình dạng ống có những ưu điểm như: diện tích bề mặt lớn, có đồng thời khả năng hấp phụ và xúc tác quang cao cũng như được chế tạo bằng các phương pháp đơn giản, tiết kiệm chi phí như: thủy nhiệt, solvat nhiệt,... [3,4]. Gần đây, graphene và vật liệu trên cơ sở graphene như graphene oxit được quan tâm đặc biệt bởi các nhà khoa học trên thế giới vì có cấu trúc hai chiều với các tính chất hóa lý vượt trội như độ linh động điện tử cao, diện tích bề mặt lớn, khả năng dẫn nhiệt, dẫn điện tốt [1]. Với những tính chất nổi bật như vậy, graphene được kỳ vọng sẽ là vật liệu tiềm năng khi lai hóa với $\mathrm{TiO}_{2}$ để tăng cường khả năng xúc tác quang. 
Trong nghiên cứu này, ống nano $\mathrm{TiO}_{2}$ được tổ hợp trong dung dịch chứa graphene dạng đĩa (nanoplatelet-sGr) bằng phương pháp thủy nhiệt đơn giản. Hình thái bề mặt được phân tích bằng ảnh hiển vi điện tử truyền qua (TEM). Cấu trúc và tính chất tinh thể của vật liệu được phân tích bằng giản đồ nhiễu xạ tia $\mathrm{X}$, phổ dao động Raman. Khả năng xúc tác quang được đánh giá thông qua sự phân hủy màu methylene xanh dưới sự chiếu sáng của ánh nắng mặt trời tự nhiên. Tính chất quang và cơ chế tăng cường khả năng xúc tác quang được giải thích thông qua phổ UVVis phản xạ rắn (DRS).

\section{Thực nghiệm}

\subsection{Hóa chất}

Hóa chất và vật liệu được sử dụng trong nghiên cứu này bao gồm: bột $\mathrm{TiO}_{2}$ thương mại (Merck), graphene nanoplatelet (sGraphene), sodium hydroxide $(\mathrm{NaOH}$, Merck), acetone $\left(\mathrm{C}_{3} \mathrm{H}_{6} \mathrm{O}\right)$, ethanol $\left(\mathrm{C}_{2} \mathrm{H}_{5} \mathrm{OH}\right)$, methylene blue (JHD Fine Chemicals, China, 99\%).

\subsection{Qui trình chế tạo vật liệu}

\subsubsection{Qui trình chế tạo ống nano $\mathrm{TiO}_{2}(\mathrm{TNT})$}

- Quá trình tạo mẫu: $34 \mathrm{~g} \mathrm{NaOH}$ cho vào cốc thủy tinh $250 \mathrm{ml}$ thêm $78 \mathrm{ml}$ nước cất, sau đó khuấy từ trong 10 phút. Tiếp đó, cho thêm 0.84 $\mathrm{g}$ bột $\mathrm{TiO}_{2}$ thương mại và khuấy 15 phút cho đến khi $\mathrm{TiO}_{2}$ phân tán đồng đều trong dung dịch. Cuối cùng dung dịch được đưa vào bình Teflon và thủy nhiệt ở nhiệt độ $135^{\circ} \mathrm{C}$ trong 24 giờ.

- Quá trình xử lí mẫu: Sau khi kết thúc quá trình thủy nhiệt, bình Teflon để nguội ở nhiệt độ phòng. Sản phẩm thu được ở dạng huyền phù, tiến hành loại bỏ phần dung dịch, thu phần bột rắn, nghiền nhỏ và cho vào cốc để lọc rửa đến $\mathrm{pH}=7$ bằng nước cất. Cuối cùng, sấy khô mẫu ở nhiệt độ $100^{\circ} \mathrm{C}$ trong 5 giờ.

2.2.2. Qui trình chế tạo vật liệu tổ hợp ống nano $\mathrm{TiO}_{2}$ với graphene (sGr/TNT)
Qui trình chế tạo vật liệu tổ hợp ống nano $\mathrm{TiO}_{2}$ và graphene cũng tương tự như quá trình chế tạo ống nano $\mathrm{TiO}_{2}$ và cũng được chia làm hai quá trình: quá trình tạo mẫu và quá trình xử lí mẫu. Tuy nhiên, trong quá trình tạo mẫu có pha thêm graphene ( $5 \%$ về khối lượng) và được khuấy từ trong 10 phút trước khi cho hỗn hợp dung dịch vào bình Teflon để tổng hợp thủy nhiệt.

\subsection{Qui trình đo hấp phu và xúc tác quang}

Để tiến hành khảo sát khả năng hấp phụ và xúc tác quang của vật liệu chúng tôi tiến hành các bước sau:

+ Pha dung dịch methylene xanh (MB) với nồng độ là 20 ppm.

+ Tiếp theo, $0.01 \mathrm{~g}$ vật liệu khảo sát cho vào cốc chứa $250 \mathrm{ml}$ dung dịch MB được khuấy đều. Ban đầu vật liệu được đặt trong buồng tối để xác định khả năng hấp phụ và thời gian hấp phụ đạt trạng thái bão hòa trước khi được chiếu sáng để đo khả năng xúc tác quang. Khoảng thời gian đo hấp phụ là: $3,6,9,12,15,20,40,60,90,120$ phút.

+ Sau khi hấp phụ đạt bão hòa, chúng tôi bắt đầu tiến hành khảo sát khả năng xúc tác quang của vật liệu dưới ánh sáng mặt trời trực tiếp từ 12 giờ trưa đến 15 giờ ở miền Nam Việt Nam, nhiệt độ khoảng $30-33^{\circ} \mathrm{C}$. Quá trình xúc tác quang được đo với các khoảng thời gian: 30, 60, 90, 120 phút sau khi được chiếu sáng. Phép đo để xác định sự suy giảm nồng độ $\mathrm{MB}$ trong quá trình hấp phụ và xúc tác quang được thực hiện bằng máy đo phổ UV-Vis DR5000 (HACH) với bước sóng trong khoảng từ 450-800 nm.

Hiệu quả của quá trình hấp phụ và xúc tác quang $(\mathrm{h})$ được tính toán bởi công thức sau:

$$
\mathrm{h}=\left(1-\frac{\mathrm{C}}{\mathrm{C}_{\mathrm{o}}}\right) \cdot 100 \%
$$

với $\mathrm{C}_{\mathrm{o}}$ là nồng độ ban đầu của $\mathrm{MB}$ trước khi hấp phụ hoặc trước khi bị phân hủy trong quá trình xúc tác quang, $C$ là nồng độ của $\mathrm{MB}$ tại mỗi thời điểm đo. 


\section{Kết quả và thảo luận}

\subsection{Hình thái bề mắt}

Hình 1 thể hiện ảnh TEM của vật liệu TNT và $\mathrm{sGr} 5 \% / \mathrm{TNT}$. Hình $1 \mathrm{a}$ cho thấy vật liệu TNT đã được chế tạo thành công với kích thước khá đồng đều, đường kính ống khoảng $6-8 \mathrm{~nm}$ và độ dài khoảng vài trăm nanomet. Đối với vật liệu tổ hợp, hình $1 \mathrm{~b}$ cho thấy sự tồn tại đồng thời ống nano $\mathrm{TiO}_{2}$ và graphene trong vật liệu này. Ngoài ra, các ống nano $\mathrm{TiO}_{2}$ đã đính trên tâm nano graphene dạng đĩa, điều này giúp cho các quang điện tử khi được kích thích dễ dàng di chuyển sang tấm nano graphene. Do đó, giúp giảm tốc độ tái hợp giữa điện tử và lỗ trống.
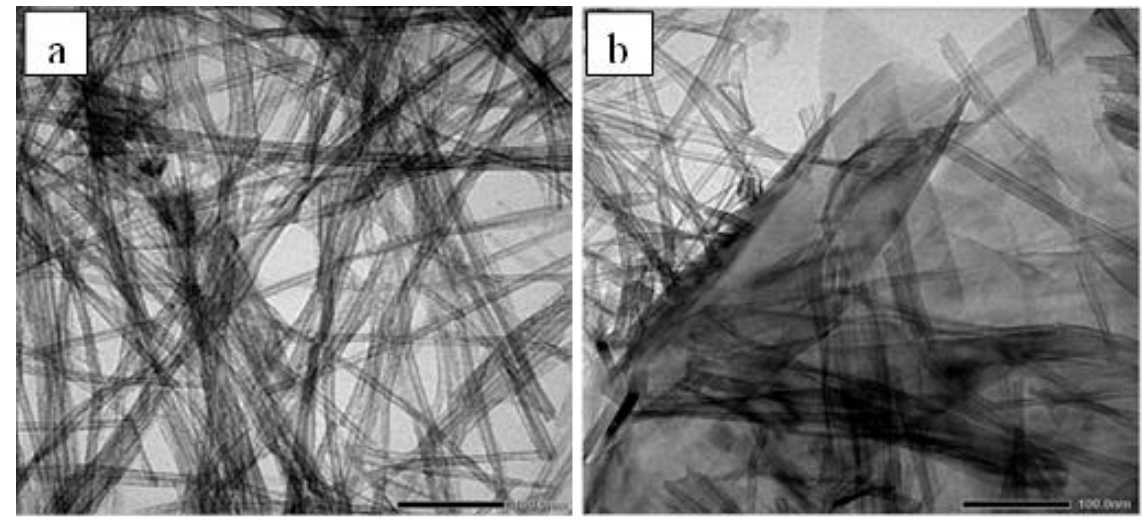

Hình 1. Hình ảnh TEM của a) TNT, và b) TNT/graphene.

\subsection{Cấu trúc tinh thể}

Hình 2 thể hiện giản đồ nhiễu $x a$ tia $X$ của vật liệu sGraphene, TNT và sGr5\%/TNT. Giản đồ XRD của sGraphene có hai đỉnh nhiễu xạ đặc trưng tại $26.18^{\circ}$ và $54.12^{\circ}$, được gán cho các mặt mang của cacbon $\mathrm{C}(002)$ và $\mathrm{C}(004)$. Trong khi đó, TNT xuất hiện các đỉnh nhiễu xạ tại $23.84^{\circ}$, $28.10^{\circ}, 47.96^{\circ}$ tương ứng cho các mặt mạng (110), (211), (020) của câu trúc monoclinic của tinh thể $\mathrm{Na}_{2-\mathrm{x}} \mathrm{H}_{2} \mathrm{Ti}_{3} \mathrm{O}_{7}$ [5] hoặc $\mathrm{Na}_{2}$ ${ }_{x} \mathrm{H}_{\mathrm{x}} \mathrm{Ti}_{2} \mathrm{O}_{4}(\mathrm{OH})_{2}(0 \leq \mathrm{x} \leq 2)$ [6]. Các nghiên cứu trước đây cho rằng khi rửa bằng axit thì xảy ra sự trao đổi ion $\mathrm{H}^{+}$với $\mathrm{Na}^{+}$và điều này làm tăng cường sự hình thành ống TNT. Cụ thể, Yang và các cộng sự của mình đã minh chứng sự phụ thuộc của tỉ lệ $\mathrm{Na}$ /Ti đối với $\mathrm{pH}$ [3], kết quả cho thấy rằng nếu $\mathrm{pH}$ càng thấp thì khả năng trao đổi ion $\mathrm{H}^{+}$và ion $\mathrm{Na}^{+}$càng cao. Trong báo cáo này, vật liệu $\mathrm{TNT}$ và $\mathrm{sGr} \% \% / \mathrm{TNT}$ được lọc rửa bằng nước cất khi giá trị $\mathrm{pH}$ đạt 7 thì vẫn còn hiện diện của nguyên tử Na trong ống nano. Trong vật liệu sGr5\%/TNT thể hiện các đỉnh đặc trưng của pha anatase ở $25.00^{\circ}, 37.24^{\circ}, 38.28^{\circ}, 53.55^{\circ}, 54.75^{\circ}$, $62.45^{\circ}$ tương ứng với các mặt mạng (101), (004),
(200), (211), (118), (116) [7-10]. Ngoài ra, vật liệu sGr5\%/TNT còn thể hiện đỉnh đặc trưng của cấu trúc graphene ở mặt mạng C(002) nhưng cường độ đỉnh đã giảm đi đáng kể, các mặt mạng (110), (211), (020) của cấu trúc ống nano TNT cũng xuất hiện với cường độ suy giảm mạnh. Bên cạnh đó, cũng có sự hiện diện hai đỉnh nhỏ của pha rutile ở $27.17^{\circ}, 36.63^{\circ}$ tương ứng với mặt mạng (110), (101) [11,12]. Các đỉnh đặc trưng của ống TNT và graphene đều giảm trong vật liệu tổ hợp sGr5\%/TNT đã chứng minh được rằng có sự tương tác giữa TNT và graphene trong quá trình thủy nhiệt.

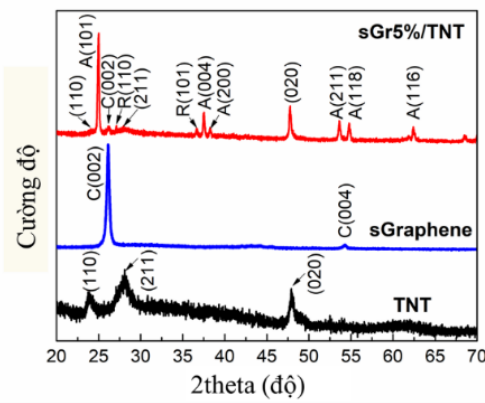

Hình 2. Giản đồ nhiễu xạ tia X (XRD) của TNT, sGraphene và sGr5\%/TNT. 


\subsection{Phổ Raman}

Hình 3 thể hiện phổ Raman của TNT, graphene và sGr5\%/TNT. Hình $3 a$, cho thấy vật liệu TNT xuất hiện các đỉnh dao động tại: 144 , 275 và $446 \mathrm{~cm}^{-1}[13,14]$. Trong đó, đỉnh $144 \mathrm{~cm}^{-1}$ thuộc về chế độ dao động Eg của pha anatase [15]. Ngoài ra, vật liệu TNT xuất hiện các dao động của cấu trúc ống với hai đỉnh nổi bật nhất tại 275 và $446 \mathrm{~cm}^{-1}$ lần lượt đặc trưng cho dao động Ti-O trong liên kết của $\mathrm{Na}-\mathrm{Ti}-\mathrm{O}$ của cấu trúc lớp và liên kết Ti-O-Ti [16]. Hơn thế nữa, vật liệu TNT còn cho thấy sự hiện diện của đỉnh dao động tại $824 \mathrm{~cm}^{-1}$ được gán cho liên kết Ti$\mathrm{O}-\mathrm{H}$ do sự thay thế ion $\mathrm{Na}^{+}$bởi $\mathrm{H}^{+}$. Đối với vật liệu sGr5\%/TNT, đỉnh dao động đặc trưng của pha anatase ở $144 \mathrm{~cm}^{-1}$ trong vật liệu ống đã dịch chuyển sang giá trị cao hơn ở $150 \mathrm{~cm}^{-1}$. Sự thay đổi blue-shifted $\left(6 \mathrm{~cm}^{-1}\right)$ có thể do sự tương tác giữa các vật liệu không đồng nhất trong vật liệu tổ hợp hoặc do sự thiếu hụt oxi [16]. Do sự liên kết của hai vật liệu này có thể làm tăng sự dịch chuyển điện tử giữa hai vật liệu và do đó cũng mang lại sự tăng cường hoạt động xúc tác quang của vật liệu. Ngoài ra, trong vật liệu tổ hợp còn có các đỉnh dao động ở $397\left(\mathrm{~B}_{1 \mathrm{~g}}\right), 512\left(\mathrm{~B}_{1 \mathrm{~g}}\right.$ và $\left.\mathrm{A}_{1 \mathrm{~g}}\right), 631\left(\mathrm{E}_{\mathrm{g}}\right) \mathrm{cm}^{-1}$ được gán cho các dao động của pha anatase $[7,18]$ và đỉnh đặc trưng của cấu trúc tại $275 \mathrm{~cm}^{-1}$. Bên cạnh đó, có sự xuất hiện dao động của liên kết $\mathrm{Ti}-\mathrm{O}-\mathrm{C}$ trong khoảng từ $670-700 \mathrm{~cm}^{-1}$ [17].

Sự hiện diện của graphene còn thể hiện trong phạm vi từ $1000-3000 \mathrm{~cm}^{-1}$. Hình $3 \mathrm{a}$ cho thấy sự hiện diện của đỉnh $\mathrm{D}$, đỉnh $\mathrm{G}$ lần lượt tại 1327 $\mathrm{cm}^{-1}, 1572 \mathrm{~cm}^{-1}$ và đỉnh $2 \mathrm{D}$ tại $2663 \mathrm{~cm}^{-1}$. Đỉnh $\mathrm{G}$ tại $1572 \mathrm{~cm}^{-1}$ đặc trưng cho dao động của cacbon lai hóa $\mathrm{sp}^{2}$, trong khi đó đỉnh $\mathrm{D}$ thể hiện dao động của các khuyết tật của cacbon lai hóa $\mathrm{sp}^{3}$. Đỉnh 2D thể hiện số lượng lớp của graphene, cường độ đỉnh $2 \mathrm{D}$ trong graphene đa lớp thì nhỏ hơn so với graphene đơn lớp.

Hơn thế, tỷ lệ cường độ $\mathrm{I}_{\mathrm{D}} / \mathrm{I}_{\mathrm{G}}$ cho biết mực độ khuyết tật của graphene [18,19]. Hình $3 b$ thể hiện tỉ lệ $\mathrm{I}_{\mathrm{D}} / \mathrm{I}_{\mathrm{G}}$ của vật liệu sGraphene và sGr5\%/TNT lần lượt là 0.44 và 0.26 . Do đó khi TNT tổ hợp với graphene đã góp phần làm giảm mức độ khuyết tật của graphene.
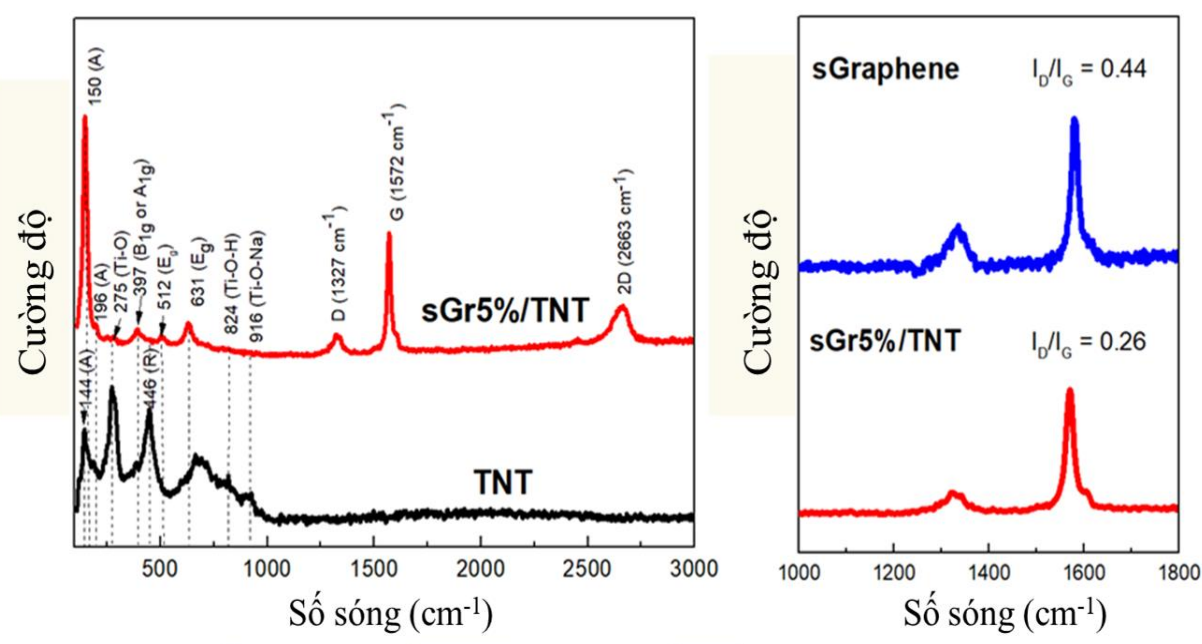

Hình 3. Phổ Raman của TNT và sGr5\%/TNT a) số sóng 100-3000 cm-1, b) số sóng 1000-1800 cm-1.

\subsection{Khả năng hấp phụ và xúc tác quang}

Thông qua các phép phân tích về hình thái học (TEM), cấu trúc tinh thể và liên kết trong các vật liệu thông qua giản đồ nhiễu xạ tia $\mathrm{X}$ và phổ
Raman đã chứng minh chế tạo thành công ống nano $\mathrm{TiO}_{2}$ (TNT) và vật liệu tổ hợp sGr5\%/TNT. Tiếp theo, tiến hành khảo sát khả năng hấp phụ và xúc tác quang của vật liệu TNT và sGr5\%/TNT. 
Hình 4 cho thấy khả năng hấp phụ và xúc tác quang của vật liệu TNT và sGr5\%/TNT. Quá trình hấp phụ đạt bão hòa sau 120 phút, kết quả cho thấy hiệu quả hấp phụ MB của TNT $66.3 \%$ tốt hơn so với sGr5\%/TNT 49.1\%. Sau khi quá trình hấp phụ đạt trạng thái cân bằng, quá trình xúc tác quang được tiếp tục dưới điều kiện chiếu sáng trực tiếp của mặt trời. Sau 120 phút chiếu sáng thì vật liệu sGr5\%/TNT đạt hiệu quả xử lý MB 95.0\% vượt trội hơn so với TNT 62.6\%. Do đó, nhờ vào sự hình thành tiếp xúc dị thể giữa ống nano $\mathrm{TiO}_{2}$ và tấm nano graphene dạng đĩa đã giúp tăng cường khả năng xúc tác quang.

3.5. Phổ phản xạ rắn và co chế tăng cuờng khả năng xúc tác quang

Hình 5 thể hiện phổ phản xạ rắn (DRS) của các vật liệu được tổng hợp. Tính chất quang của vật liệu TNT và vật liệu tổ hợp sGr5\%/TNT cho thấy sự hấp thụ tốt trong vùng UV là do sự hấp thụ năng lượng photon của $\mathrm{TiO}_{2}$ kích thích các điện tử từ vùng hóa trị sang vùng dẫn $\left(\mathrm{O}_{2 \mathrm{p}} \rightarrow\right.$ $\mathrm{Ti}_{3 \mathrm{~d}}$ ) [20]. Hình 5 cho thấy vật liệu sGr5\%/TNT có khả năng hấp thụ ánh sáng nhìn thấy tốt hơn so với vật liệu TNT. Sự dịch chuyển khả năng hấp thụ sang vùng bước sóng dài hơn đối với vật liệu sGr5\%/TNT đã cho thấy sự thu hẹp năng lượng vùng cấm của vật liệu này [20]. Từ dữ liệu của phổ DRS, có thể xác định giá trị năng lượng vùng cấm bằng cách dùng hàm Kubelka-Munk theo phương pháp Tauc. Theo các nghiên cứu trước đây cho thấy giá trị năng lượng vùng cấm pha anatase của $\mathrm{TiO}_{2}$ là $\sim 3.2 \mathrm{eV}$ và đối với ống nano $\mathrm{TiO}_{2}$ là $\sim 3.7 \mathrm{eV}[21,22]$. Trong nghiên cứu này, bằng phương pháp Tauc được thể hiện trong hình 6 đã xác định được năng lượng vùng cấm của $\mathrm{TNT}$ và $\mathrm{sGr} 5 \% / \mathrm{TNT}$ lần lượt là $3.68 \mathrm{eV}$ và $3.25 \mathrm{eV}$. Do đó, nhờ vào sự tổ hợp giữa ống nano với tấm nano graphene đã làm suy giảm năng lượng vùng cấm. Ngoài ra, thông qua liên kết hóa học $\mathrm{Ti}-\mathrm{O}-\mathrm{C}$ giữa các ống nano $\mathrm{TiO}_{2}$ và các tấm nano graphene tạo điều kiện thuận lợi cho các điện tử di chuyển từ ống nano $\mathrm{TiO}_{2}$ sang graphene, làm giảm sự tổ hợp của các điện tử và lỗ trống $[11,23]$. Nhờ vậy, thời gian sống của điện tử được kéo dài giúp tăng cường phản ứng phân hủy chất màu hữu cơ $\mathrm{MB}$ và cải thiện khả năng xúc tác quang.
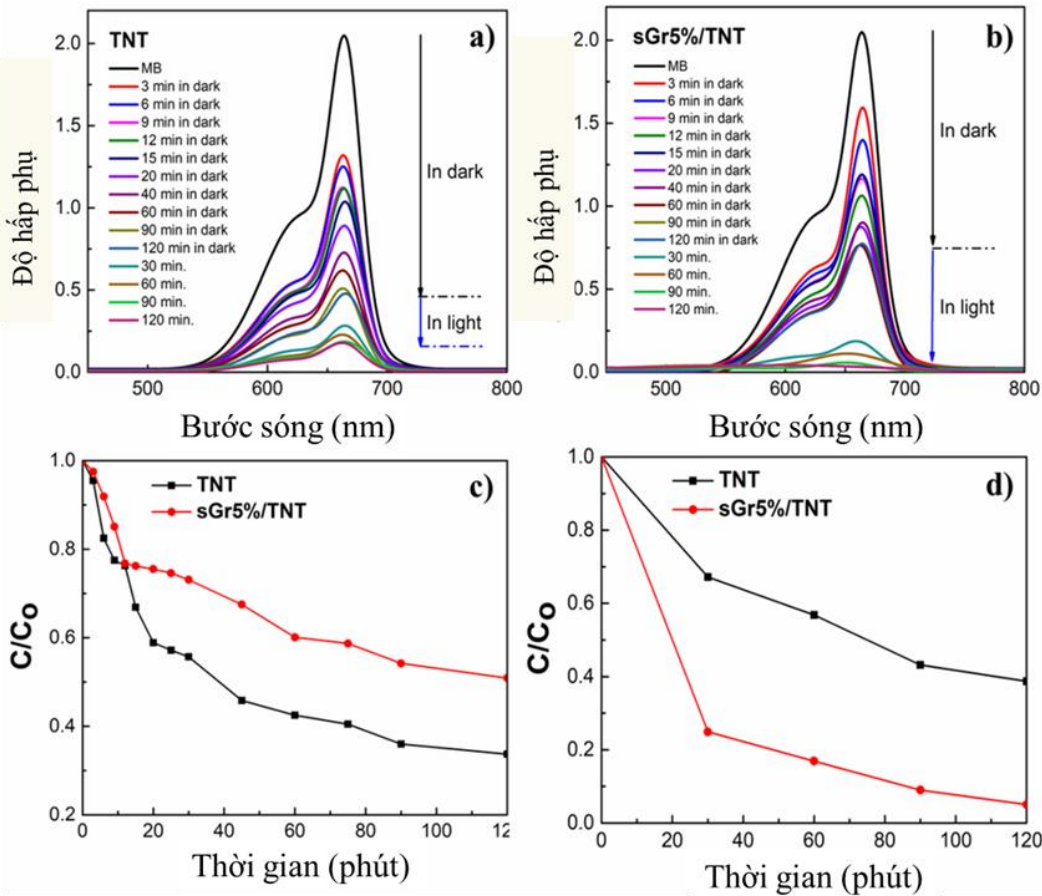

Hình 4. a), b) Biểu đồ thể hiện sự suy giảm nồng độ MB của TNT và sGr5\%/TNT và c), d) Khả năng hấp phụ và xúc tác quang của TNT và sGr5\%/TNT. 


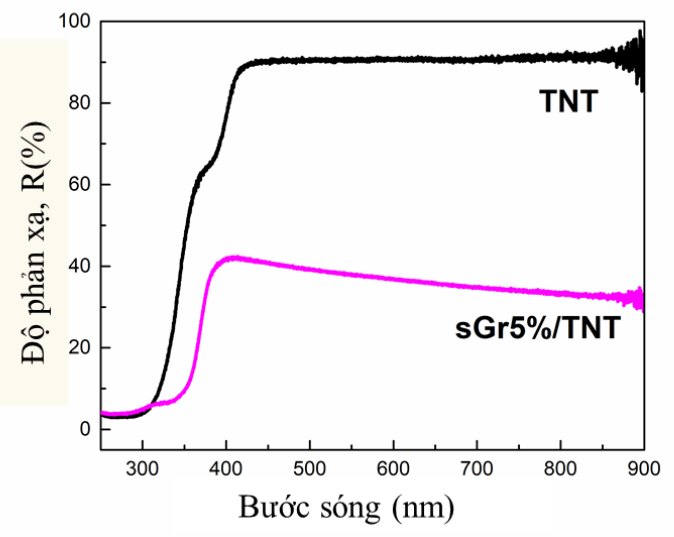

Hình 5. Phổ UV-Vis phản xạ rắn (DRS).
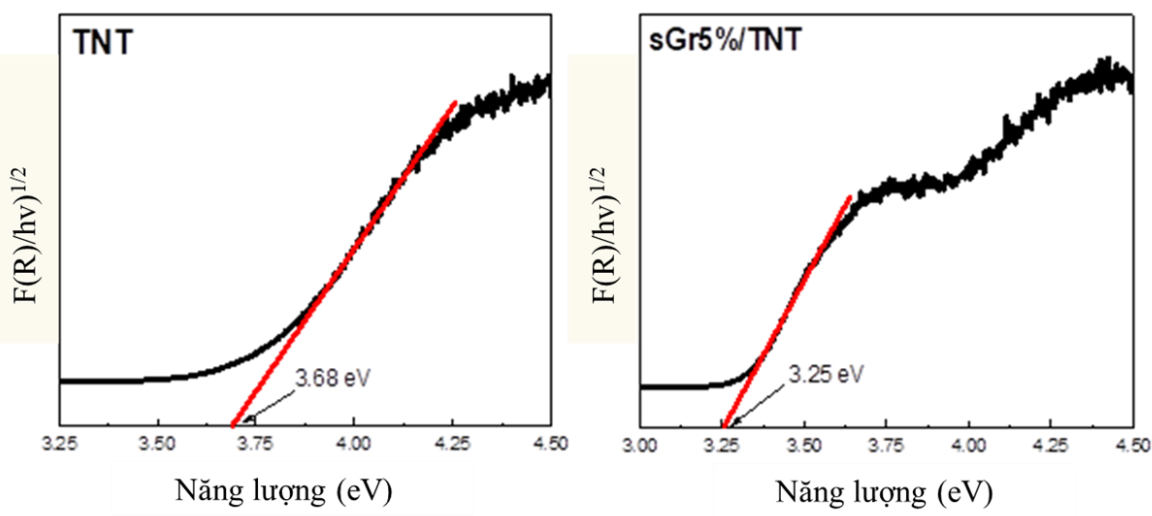

Hình 6. Năng lượng vùng cấm tính theo hàm Kubelka-Munk.

\section{Kết luận}

Trong nghiên cứu này, chúng tôi đã chế tạo thành công vật liệu TNT với đường kính đồng đều $~ 8 \mathrm{~nm}$ và vật liệu tổ hợp sGr5\%/TNT được minh chứng thông qua các phép phân tích về hình thái học (TEM), giản đồ nhiễu xạ tia $\mathrm{X}$ (XRD), phổ Raman. Ngoài ra, phổ Raman cho thấy dao động ở số sóng thấp nhất $\left(\mathrm{E}_{\mathrm{g}}\right)$ bị dịch chuyển $\sim 6 \mathrm{~cm}^{-1}$ từ $144 \mathrm{~cm}^{-1}$ sang $150 \mathrm{~cm}^{-1}$ là do sự hình thành tiếp xúc dị thể giữa TNT và graphene cũng như dao động của liên kết hóa học Ti-O-C trong khoảng từ $670-700 \mathrm{~cm}^{-1}$. Bên cạnh đó, vật liệu tổ hợp vật liệu ống nano $\mathrm{TiO}_{2}$ với tấm nano graphene dạng đĩa đã giúp tăng cường khả năng xúc tác quang phân hủy chất màu hữu cơ MB dưới điều kiện chiếu sáng của mặt trời. Cơ chế tăng cường khả năng xúc tác quang của vật liệu tổ hợp được giải thích thông qua phổ phản xạ rắn (DRS), vật liệu sGr5\%/TNT có khả năng hấp thụ ánh sáng nhìn thấy tốt hơn và có năng lượng vùng cấm thấp hơn $\mathrm{TNT}$.

\section{Lời cảm ơn}

Nghiên cứu này được tài trợ bởi Quỹ Phát triển khoa học và công nghệ Quốc gia (NAFOSTED) trong đề tài mã số 103.022019.362.

Nhóm tác giả trân trọng cảm ơn Võ Cao Minh đã đọc và chỉnh sửa bản thảo bài báo. 


\section{Tài liệu tham khảo}

[1] B. Tang, H. Chen, H. Peng, Z. Wang, W. Huang, Graphene modified $\mathrm{TiO}_{2}$ composite photocatalysts: mechanism, progress and perspective, Nanomaterials 8 (2018) 105. https:// doi.org/10.3390/nano8020105.

[2] V. Etacheri, C. Di Valentin, J. Schneider, D. Bahnemann, S.C. Pillai, Visible-light activation of $\mathrm{TiO}_{2}$ photocatalysts: Advances in theory and experiments, Journal of Photochemistry and Photobiology C: Photochemistry Reviews 25 (2015) 1-29. https://doi.org/10.1016/j.jphotochemrev.2015. 08.003 .

[3] H.H. Ou, S.L. Lo, Review of titania nanotubes synthesized via the hydrothermal treatment: Fabrication, modification, and application, Separation and Purification Technology 58 (2007)179-191. https://doi.org/10.1016/j.seppur. 2007.07.017.

[4] N. Liu, X. Chen, J. Zhang, J.W. Schwank, A review on $\mathrm{TiO}_{2}$-based nanotubes synthesized via hydrothermal method: Formation mechanism, structure modification, and photocatalytic applications, Catalysis Today 225 (2014) 34-51. https://doi.org/10.1016/j.cattod.2013.10.090.

[5] Q. Chen, G. Du, S. Zhang, L.-M. Peng, The structure of trititanate nanotubes, Acta Crystallographica Section B: Structural Science 58(2002)587-593. https://doi.org/10.1107/S0108 768102009084 .

[6] J. Yang, Z. Jin, X. Wang, W. Li, J. Zhang, S. Zhang, X. Guo, Z. Zhang, Study on composition, structure and formation process of nanotube $\mathrm{Na}_{2} \mathrm{Ti}_{2} \mathrm{O}_{4}(\mathrm{OH})_{2}$, Dalton Transactions (2003)38983901. https://doi.org/10.1039/B305585J.

[7] S.D. Perera, R.G. Mariano, K. Vu, N. Nour, O. Seitz, Y. Chabal, K.J. Balkus Jr, Hydrothermal synthesis of graphene-TiO2 nanotube composites with enhanced photocatalytic activity, ACS Catalysis 2 (2012) 949-956. https://doi.org/10. $1021 / \mathrm{cs} 200621 \mathrm{c}$.

[8] X. Zhang, B. Zhang, D. Huang, H. Yuan, M. Wang, Y. Shen, $\mathrm{TiO}_{2}$ nanotubes modified with electrochemically reduced graphene oxide for photoelectrochemical water splitting, Carbon 80 (2014)591-598. https://doi.org/10.1016/j.carbon. 2014.09.002.

[9] H. Tao, X. Liang, Q. Zhang, C.-T. Chang, Enhanced photoactivity of graphene/titanium dioxide nanotubes for removal of Acetaminophen, Applied Surface Science 324
(2015)258-264. https://doi.org/10.1016/j.apsusc. 2014.10.129.

[10] Y. Zhang, Z.-R. Tang, X. Fu, Y.-J. Xu, TiO ${ }_{2}-$ graphene nanocomposites for gas-phase photocatalytic degradation of volatile aromatic pollutant: is $\mathrm{TiO}_{2}-$ graphene truly different from other $\mathrm{TiO}_{2}-$ carbon composite materials?, ACS Nano 4 (2010) 7303-7314. https://doi.org/10. 1021/nn1024219.

[11] T. Kasuga, M. Hiramatsu, A. Hoson, T. Sekino, K. Niihara, Titania nanotubes prepared by chemical processing, Advanced Materials 11 (1999)1307-1311. https://doi.org/10.1002/(SICI) 1521-4095(199910)11:15<1307::AIDADMA1307>3.0.CO;2-H

[12] X. Pan, Y. Zhao, S. Liu, C.L. Korzeniewski, S. Wang, Z. Fan, Comparing graphene- $\mathrm{TiO}_{2}$ nanowire and graphene- $\mathrm{TiO}_{2}$ nanoparticle composite photocatalysts, ACS applied materials \& interfaces 4 (2012) 3944-3950. https://doi.org/ 10.1021/am300772t.

[13] F. Hardcastle, Raman spectroscopy of titania $\left(\mathrm{TiO}_{2}\right)$ nanotubular water-splitting catalysts, Journal of the Arkansas academy of science 65 (2011) 43-48.

[14] F. Jiang, S. Zheng, L. An, H. Chen, Effect of calcination temperature on the adsorption and photocatalytic activity of hydrothermally synthesized $\mathrm{TiO}_{2}$ nanotubes, Applied Surface Science 258 (2012) 7188-7194. https://doi.org/10. 1016/j.apsusc.2012.04.032.

[15] U. Balachandran, N. Eror, Raman spectra of titanium dioxide, Journal of Solid State Chemistry 42 (1982) 276-282.

[16] L. Qian, Z.-L. Du, S.-Y. Yang, Z.-S. Jin, Raman study of titania nanotube by soft chemical process, Journal of Molecular Structure 749 (2005)103107. https://doi.org/10.1016/j.molstruc.2005.04. 002.

[17] S.X. Nguyen, T.T. Tung, P.T.L. Huong, N.H. Tho, D. Losic, Heterojunction of graphene and titanium dioxide nanotube composites for enhancing photocatalytic activity, Journal of Physics D: Applied Physics 51 (2018) 265304. https://doi.org/10.1088/1361-6463/aac7ce.

[18] L.C. Sim, K.H. Leong, S. Ibrahim, P. Saravanan, Graphene oxide and $\mathrm{Ag}$ engulfed $\mathrm{TiO}_{2}$ nanotube arrays for enhanced electron mobility and visiblelight-driven photocatalytic performance, Journal of Materials Chemistry A 2 (2014) 5315-5322. https://doi.org/10.1039/C3TA14857B. 
[19] P. Song, X. Zhang, M. Sun, X. Cui, Y. Lin, Graphene oxide modified $\mathrm{TiO}_{2}$ nanotube arrays: enhanced visible light photoelectrochemical properties, Nanoscale 4 (2012) 1800-1804. https://doi.org/10.1039/C2NR11938B.

[20] Q. Huang, S. Tian, D. Zeng, X. Wang, W. Song, Y. Li, W. Xiao, C. Xie, Enhanced photocatalytic activity of chemically bonded $\mathrm{TiO}_{2}$ /graphene composites based on the effective interfacial charge transfer through the $\mathrm{C}-\mathrm{Ti}$ bond, Acs Catalysis 3 (2013) 1477-1485. https://doi.org/10. $1021 / \operatorname{cs} 400080 \mathrm{w}$.

[21] Q. Zhang, Y. Li, E.A. Ackerman, M. Gajdardziska-Josifovska, H. Li, Visible light responsive iodine-doped $\mathrm{TiO}_{2}$ for photocatalytic reduction of $\mathrm{CO}_{2}$ to fuels, Applied Catalysis A: General 400 (2011) 195-202. https://doi.org/10. 1016/j.apcata.2011.04.032.

[22] D.V. Bavykin, S.N. Gordeev, A.V. Moskalenko, A.A. Lapkin, F.C. Walsh, Apparent twodimensional behavior of $\mathrm{TiO}_{2}$ nanotubes revealed by light absorption and luminescence, The Journal of Physical Chemistry B 109 (2005) 8565-8569. https://doi.org/10.1021/jp050762m.

[23] W. Fang, M. Xing, J. Zhang, Modifications on reduced titanium dioxide photocatalysts: A review, Journal of Photochemistry and Photobiology C: Photochemistry Reviews 32 (2017)21-39. https://doi.org/10.1016/j.jphoto chemrev.2017.05.003. 